\title{
VIEKIRA PAK associated drug-induced interstitial lung disease: Case series with systematic review of literature
}

\author{
Yu Jun Wong ${ }^{1 *}$, Si Yuan Chew ${ }^{2 *}$, John Chen Hsiang ${ }^{1}$, Prem Harichander Thurairajah', Rahul Kumar ${ }^{1}$, Eng Kiong Teo', \\ Roshni Sadashiv Gokhale ${ }^{2}$, Imran Bin Mohamed Noor ${ }^{2}$, and Jessica Tan ${ }^{1}$
}

'Department of Gastroenterology and Hepatology, Changi General Hospital, Singapore; ${ }^{2}$ Department of Respiratory Medicine and Critical Care, Changi General Hospital, Singapore, Singapore

Dear Editor,

VIEKIRA PAK (ritonavir-boosted paritavir/ombitasvir and dasabuvir) is an effective direct-antiviral agent (DAA) licensed for patients with genotype 1 hepatitis C virus (HCV) infection. There have been emerging post-marketing reports of VIEKIRA PAK associated with drug-induced lung disease. Drug-induced interstitial lung disease (DILD) is a rare, yet potentially life-threatening complication of chronic HCV treatment. We report a case series of two patients who required mechanical ventilation for acute respiratory failure from severe VIEKIRA PAK DILD and systematically review the literature on VIEKIRA PAK associated DILD.

\section{CASE 1}

A 56-year old man was diagnosed with chronic HCV infection since January 2017 (Genotype 1A) with advanced fibrosis on transient elastography (13.3 kPa). The likely etiology of HCV infection was attributed to prior practice of intravenous drug abuse. His latest abdominal ultrasound scan showed no evidence of hepatocellular carcinoma. There was no hepatitis B or human immunodeficiency virus (HIV) co-infection. He was an ex-smoker of 20 pack years and had quit smoking 3 years ago. He was treatmentnaïve, and was initiated on VIEKIRA PAK and ribavirin from 9 May 2017 with a pretreatment HCV RNA viral load of $6.9 \log \mathrm{IU} / \mathrm{mL}$. One week after initiation of VIEKIRA PAK, he was admitted to the intensive care unit (ICU) for sudden onset of dyspnea, pleuritic chest pain and fever. Chest radiography showed bilateral air space consolidation with bilateral pleural effusions (Fig. 1A). Arterial blood gas sampling performed on supplemental oxygen administered via a non-rebreather facemask showed severe type 1 respiratory failure with a pH 7.44, $\mathrm{PaO}_{2} 64 \mathrm{mmHg}, \mathrm{PaCO}_{2} 30 \mathrm{mmHg}$ and $\mathrm{SaO}_{2} 93 \%$. He was intubated and started on mechanical ventilation.

He was commenced on empiric antibiotic therapy with intravenous meropenem, azithromycin and oseltamivir. Computed tomography (CT) scan of the thorax showed confluent air-space opacities of the bilateral upper lobes and right middle lobes (Fig. 1B). Autoimmune serologies were negative except for antinuclear antibodies with a titre of 1:80 with no discernible pattern. Bronchoalveolar lavage (BAL) showed alveolar macrophages with mixed neutrophil and lymphocyte infiltrates without malignant cells. All blood, endotracheal aspirate and BAL samples for bacterial, fungal, acid fast bacilli (AFB) cultures and respiratory virus multiplex

\footnotetext{
Abbreviations:

AFB, acid fast bacilli; BAL, bronchoalveolar lavage; CT, computer tomography: DAA, direct-antiviral agent; DILD, drug-induced interstitial lung disease; DLCO, carbon monoxide diffusing capacity; FEV ${ }_{1}$, forced expiratory volume in 1 second; FVC, full vital capacity; HARVONI, ledipasvir 90 mg/sofosbuvir 400 mg; $\mathrm{HCV}$, hepatitis $\mathrm{C}$ virus; HIV, human immunodeficiency virus; ICU, intensive care unit; ILD, interstitial lung disease; PCR, polymerase chain reaction; VIEKIRA PAK, ritonavir-boosted paritavir/ombitasvir and dasabuvir
}

\section{Corresponding author: Yu Jun Wong}

Department of Gastroenterology and Hepatology, Changi General Hospital, 2 Simei Street 3, Singapore, 529889, Singapore

Tel: $+65-68503558$

E-mail: eugene.wong.y.j@singhealth.com.sg

https://orcid.org/0000-0002-0727-1183 

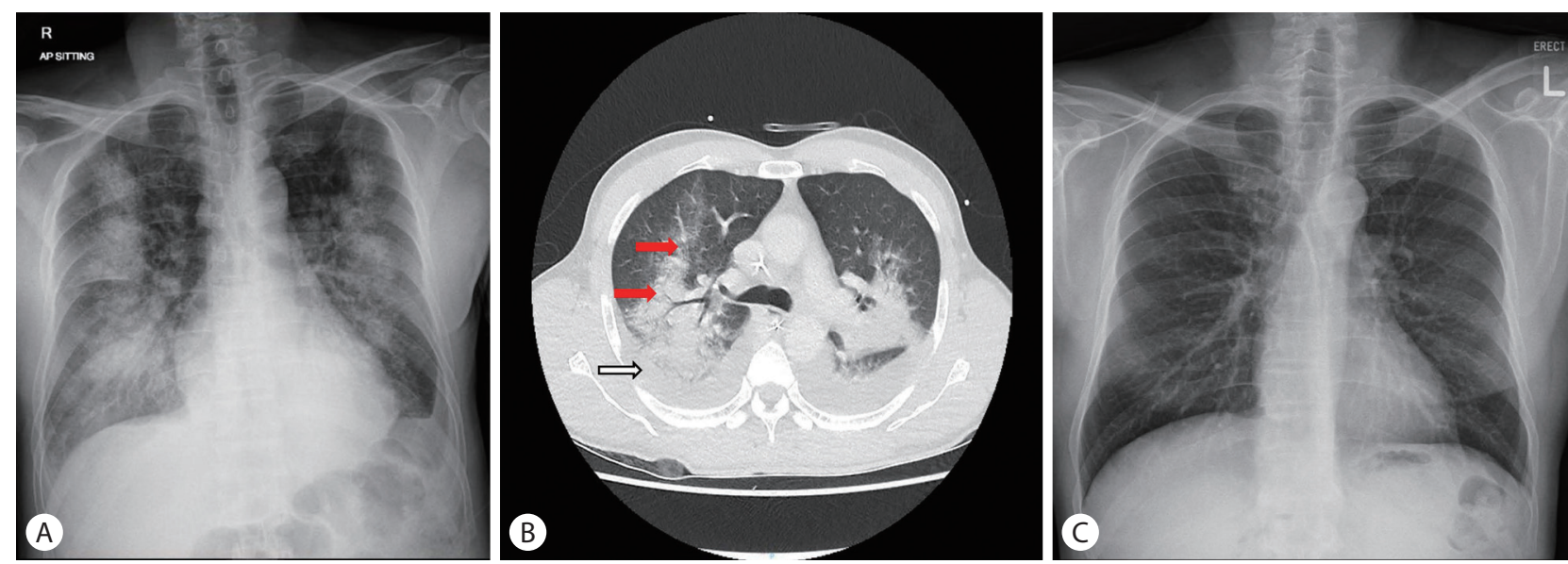

Figure 1. Radiological findings in patient 1. Chest radiograph on admission showing bilateral air space consolidation with bilateral small pleural effusion (A). Computed tomography of chest showed confluent air-space opacities with ground-glass appearance seen in bilateral upper and middle lobe (red arrows) with bilateral pleural effusion (white arrow) in transverse view (B). Repeated chest radiograph with complete resolution on day 69 (C).

polymerase chain reaction (PCR) were negative. Given the absence of infective etiology after extensive work-up, a provisional diagnosis of DILD was made. VIEKIRA PAK were withheld since admission and patient received three days of intravenous methylprednisolone $500 \mathrm{mg}$ daily followed by intravenous hydrocortisone $100 \mathrm{mg} 8$ hourly for another three days. He responded to above treatment, extubated on day 6 of admission and was discharged from hospital on day 15 of admission with a tailing regimen of oral prednisolone starting with $30 \mathrm{mg}$ daily tapered over 12 weeks with a stepwise dose reduction of $5 \mathrm{mg}$ every 2 weeks. He remained well during his review in the outpatient respiratory clinic on day 69 of illness with complete radiological resolution of air-space opacities on his chest radiograph (Fig. 1C). He was subsequently treated with HARVONI (ledipasvir $90 \mathrm{mg} /$ sofosbuvir $400 \mathrm{mg}$ ) for 12 weeks and has tolerated the treatment well without adverse reaction.

\section{CASE 2}

A 42-year old ex-smoker with treatment-naïve, compensated HCV Child's A cirrhosis (Genotype 1A) and cardiac varices on surveillance gastroscopy was commenced on VIEKIRA PAK and ribavirin with a pre-treatment HCV RNA load of $5.19 \log \mathrm{IU} / \mathrm{mL}$. He developed a sore throat and non-productive cough 20 days after initiation of treatment. The initial chest radiograph performed was unremarkable. He was admitted 20 days later (Day 40 of VIEKIRA PAK) for worsening dyspnea. His vital signs upon presentation were: temperature of 37.0 , blood pressure $104 / 66 \mathrm{mmHg}$, heart rate $67 \mathrm{bpm}$ and oxygen saturation of $92 \%$ on room air. Initial chest radiograph on admission showing bilateral air-space opacities in bilateral upper zones (Fig. 2A). He was admitted to the general ward and commenced on empiric intravenous Ceftriaxone and oral clarithromycin for presumptive pneumonia. However, apart from a positive respiratory virus PCR test for rhinovirus on a nasopharyngeal swab specimen, his blood, sputum and urine samples for bacterial, fungal and AFB were all negative. CT thorax on day 4 of admission showed bilateral symmetrical ground-glass opacities with associated smooth interlobular septal thickening and absence of pulmonary embolism (Fig. 2B). As he continued to deteriorate with worsening hypoxemia, VIEKIRA PAK was held off and he was transferred to the ICU on day 10 of admission and intubated the next day. BAL contained a mixture of neutrophils $(50 \%)$, alveolar macrophages (25\%), lymphocytes (15\%) and eosinophils (10\%) without malignant cells. BAL samples for respiratory virus isolation, bacterial culture, galactomannan, pneumocystis jiroveci PCR were all negative. Although his antinuclear antibody titre were elevated at 1:160 (speckled pattern), the remaining autoimmune serologies were negative. A provisional diagnosis of DILD was made based on: (1) the temporal relationship of his presentation with the initiation of VIEKIRA PAK, (2) lack of response to empiric antibiotic therapy, and (3) absence of positive microbiological or autoimmune tests to explain an alternative etiology. He was treated with intravenous methylprednisolone $500 \mathrm{mg}$ daily for 3 days from day 12 of admission and was extubated successfully on day 15 of admission. This was followed by a tapering dose of oral prednisolone over 2 months. A repeat CT scan of the thorax was performed on day 16 of admission which showed im- 
provement in ground glass changes in both upper, middle and lingula lobes. Thickened interlobular and intralobular septal were still present and seen to extend to involve the lower lobes now with architectural distortion and mild traction bronchiectasis. He remained dependent on supplemental oxygen until day 27 and was discharged on day 44 of admission after undergoing a period of inpatient rehabilitation. Lung function testing on day 51 since admission showed a moderate restrictive defect with a forced expiratory volume in 1 second $\left(\mathrm{FEV}_{1}\right)$ over full vital capacity (FVC) ratio of $75 \%, \mathrm{FEV}_{1} 1.89 \mathrm{~L}$ (67\% predicted), FVC $2.52 \mathrm{~L}$ (63\% predicted), total lung capacity of $3.97 \mathrm{~L}(73 \%$ predicted), and severe reduction in diffusion capacity with carbon monoxide diffusing capacity (DLCO) (corrected for hemoglobin) value of $29 \%$ predicted. He was reviewed in the outpatient clinic on day 209 with near complete resolution of symptoms of dyspnea and interstitial opacities on chest radiography (Fig. 2C), even though his diffusion capacity remained severely reduced with DLCO value of $29 \%$ predicted on repeated lung function test performed on day 151 . We hypothesize that the more severe clinical course of this patient could be attributed to the delayed cessation of VIEKIRA PAK which was only stopped on day 10 of admission.

\section{DISCUSSION}

VIEKIRA PAK is a fixed-dose combination capsule comprising 3 different DAAs which target different sites on the HCV genome, namely Paritaprevir (NS3 protease inhibitor), Ombitasvir (NS5A inhibitor), and Dasabuvir (NS5B inhibitor). The role of ritonavir, a CYP3A inhibitor with no anti HCV activity, in VIEKIRA PAK is to increase the half-life of Paritaprevir which is primarily metabolized via CYP3A4 pathway. VIEKIRA PAK has excellent efficacy among genotype $1 \mathrm{HCV}$ patients. Eight randomized multicenter phase III trials involving 2534 genotype $1 \mathrm{HCV}$ patients showed consistent sustained virological response (SVR) of $>95 \%$ including patients with liver cirrhosis, prior treatment and post liver transplantation. $^{1-4}$ Currently, VIEKIRA PAK has been accepted for treatment of HCV patients by European and American guidelines. ${ }^{5,6}$ VIEKIRA PAK is generally safe and well tolerated, with headache, fatigue, nausea, pruritus and insomnia (>10\% of subjects) being the commonest adverse events. ${ }^{7}$ Only liver decompensation and angioedema had been reported in post-marketing surveillance by $\mathrm{Ab}$ bie. $^{8}$

The introduction of DAA has revolutionized the landscape of HCV treatment because of its high clinical efficacy in achieving sustained virological response and better tolerability. With increasing use of DAAs, pulmonary complications have also been reported following DAA therapies when used in combination with pegylated interferon or as monotherapy. ${ }^{9-11}$ Although DILD is a well-recognized complication of pegylated interferon and ribavirin combination therapy, ${ }^{12,13}$ there have been no documented cases of serious pulmonary toxicity with ribavirin therapy alone. This has
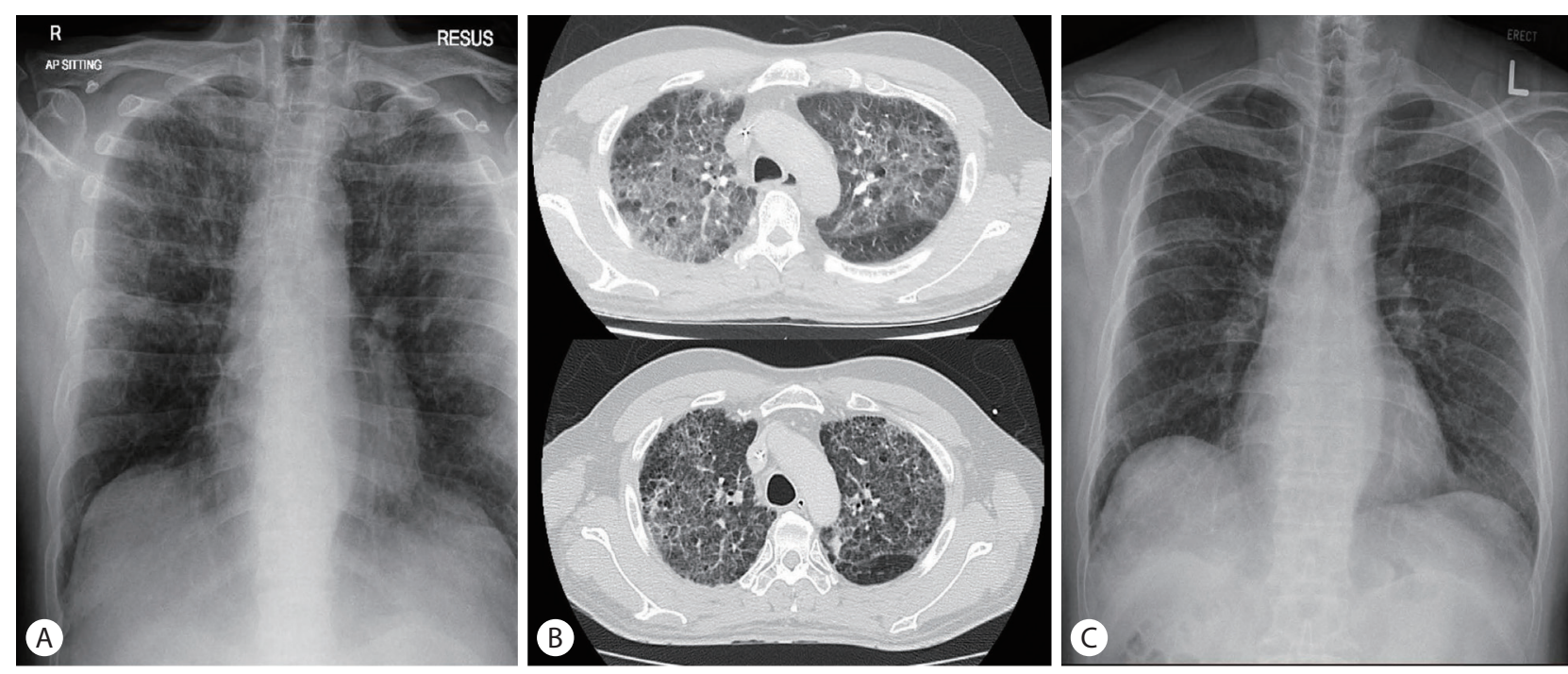

Figure 2. Radiological findings in patient 2. Chest radiograph on admission showing bilateral air-space opacities in bilateral upper zones (A). Computed tomography scan of the chest showed bilateral symmetrical ground-glass opacities with associated smooth interlobular septal thickening involving the upper zone sparing the lung bases during acute (upper) and recovery phase (lower) (B). Repeat chest radiograph with near complete resolution on day 209 (C). 
Yu Jun Wong, et al.

given rise to the hypothesis that interferon is the etiological agent responsible. To our knowledge, this is the first case series of VIEKIRA PAK associated DILD reported.

In our case series, both patients had grade 4 pneumonitis under Common Terminology Criteria for Adverse Events (CTCAE-v4.03) as they both require intubation and mechanical ventilation for respiratory failure. While both of our patients did not have baseline pulmonary function testing or testing of the presence of HCV-RNA in $B A L$, they did not have any respiratory symptoms prior to VIEKIRA PAK therapy. Both patients demonstrated rapid respiratory failure with subsequent complete resolution of symptoms and radiological changes after cessation of VIEKIRA PAK therapy. As the anti-HCV therapy for both patients was withheld until complete resolution of respiratory symptoms, one would expect them to have progression of interstitial lung disease (ILD) if it was due to chronic HCV infection. We therefore believe that this hyperacute temporal sequence is more in keeping with drug-related ILD as opposed to chronic HCV infection related ILD, where one would expect patients with the latter condition to have more subacute course with progressive deterioration. Although both patients received ribavirin on top of VIEKIRA PAK, there were no significant drug-drug interaction identified. Patient in case 1 had no other medication while the patient in case 2 was only received propranolol and omeprazole.

We performed a systematic review of the literature and only two cases of VIEKIRA PAK associated DILD have been reported to date. $^{14,15}$ The clinical features of all patients with VIEKIRA PAK associated DILD (including our two patients from our case series) are summarized in Table 1. Wu et al described a 68-year-old Chinese woman who presented with respiratory distress one week into treatment with VIEKIRA PAK without ribavirin for genotype 1b HCV infection. She made a prompt and complete recovery after cessation of VIEKIRA PAK. However when VIEKIRA PAK was reintroduced 3 weeks later the patient was readmitted for respiratory failure requiring mechanical ventilation for respiratory failure and high dose corticosteroid therapy. ${ }^{14}$ Tarao et al. reported a 77-year-old Japanese woman who reported fatigue 4 weeks into treatment with VIEKIRA PAK only for genotype $1 \mathrm{~b}$ HCV infection. VIEKIRA PAK was discontinued after 7 weeks' duration but she developed a dry cough 10 days afterwards and was subsequently diagnosed with interstitial pneumonia on further laboratory and radiological testing. ${ }^{15}$ She had resolution of interstitial pneumonia without requiring mechanical ventilation or corticosteroid therapy. ${ }^{15}$ Based on the clinical experience of our case series and case reports, it appears that the most important prognostic factor

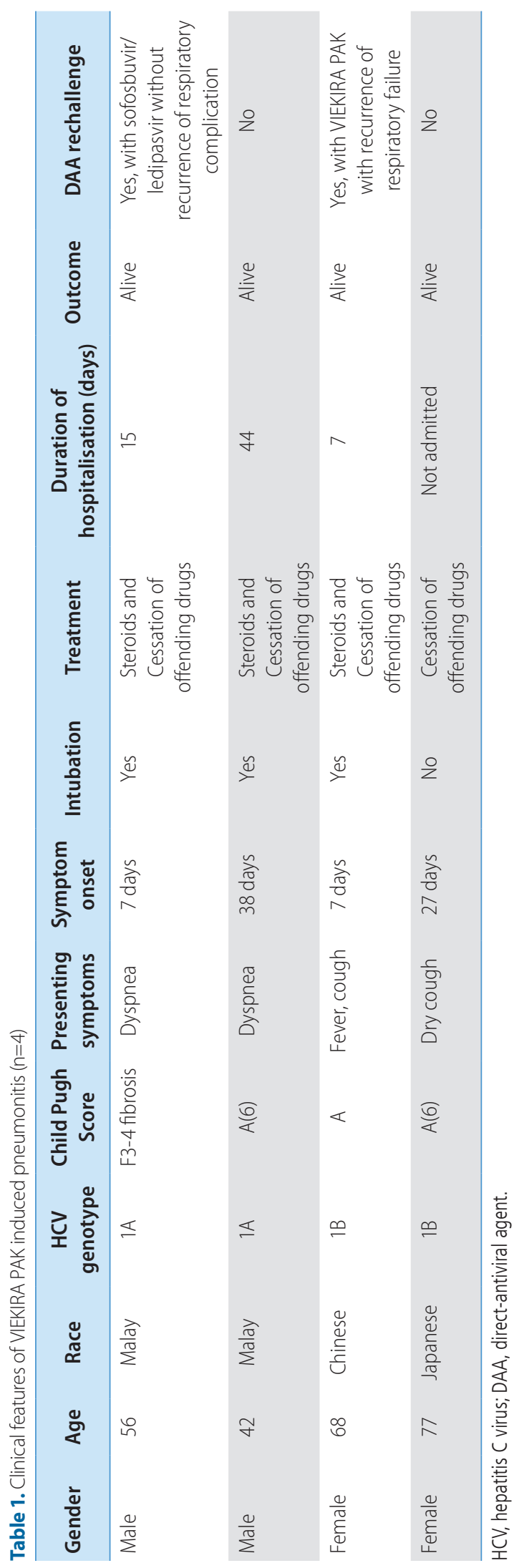


when VIEKIRA PAK DILD has occurred is the early recognition and cessation of VIEKIRA PAK. We observe that the clinical presentation of VIEKIRA PAK DILD can resemble that of pneumonia and this may lead to delayed cessation of VIEKIRA PAK or even rechallenge of VIEKIRA PAK after initial recovery. Physicians should therefore have greater awareness of this rare but potentially lifethreatening adverse reaction and consider early discontinuation of VIEKIRA PAK therapy when patients present with a pneumonialike syndrome soon after initiation of VIEKIRA PAK therapy.

To date, VIEKIRA PAK associated DILD has only been reported among Asian HCV patients. Such findings have never reported in the earlier landmark trials, which recruited predominantly Caucasian patients. Genetic predisposition may be a contributory factor, however further prospective controlled studies are required to conclude such finding. Based on limited literature from our case series it is very difficult to determine the ingredient responsible for DILD. Ritonavir was used in HIV patients since 1996 and was appeared to be safe. One patient was re-treated and completed 12 weeks of HARVONI without any respiratory complication. Because this patient had tolerated NS5A and NS5B containing HARVONI, we postulate that paritaprevir, the protease inhibitor is probably the ingredient responsible for DILD. It is possible that cirrhotic patients may be more susceptible to the ritonavir boosted protease inhibitors therapy. Such patients should be managed vigilantly by specialist with sufficient experience with Hepatitis $\mathrm{C}$ because early recognition could be potentially lifesaving.

In summary, VIEKIRA PAK associated DILD is a rare yet potentially life-threatening complication that has so far been only reported in Asian patients. As DAA is expected to replace pegylated interferon as the future of HCV treatment, stringent post marketing surveillance is needed to better understand the risks of DILD with VIEKIRA PAK therapy. Physicians should have a heightened awareness of the possibility of DILD when patients report respiratory symptoms after initiating therapy with VIEKIRA PAK as the delayed cessation of VIEKIRA PAK may result in worse outcomes.

\section{Author's contribution}

WYJ and CSY performed data collection and drafted the manuscript. All authors critically reviewed the manuscript and approved the final draft.

\section{Conflicts of Interest}

The authors have no conflicts to disclose.

\section{REFERENCES}

1. Ferenci $P$, Bernstein D, Lalezari J, Cohen D, Luo Y, Cooper $C$, et al. ABT-450/r-ombitasvir and dasabuvir with or without ribavarin for HCV. N Engl J Med 2014;370:1983-1992.

2. Feld JJ, Kowdley KV, Coakley E, Sigal S, Nelson DR, Crawford D, et al. Treatment of HCV with ABT-450/r-ombitasvir and dasabuvir with ribavarin. N Engl J Med 2014;370:1594-1603.

3. Poordad F, Hezode C, Trinh R, Kowdley KV, Zeuzem S, Agarwal K, et al. ABT-450/r-ombitasvir and dasabuvir with ribavirin for hepatitis $C$ with cirrhosis. N Engl J Med 2014;370:1973-1982.

4. Zeuzem S, Jacobson IM, Baykal T, Marinho RT, Poordad F, Bourlière $M$, et al. Retreatment of HCV with ABT-450/r-ombitasvir and dasabuvir with ribavarin. N Engl J Med 2014;370:1604-1614.

5. European Association for the Study of the Liver. EASL recommendation on treatment of hepatitis C 2016. J Hepatol 2017;66:153-194.

6. AASLD/IDSA HCV Guidance Panel. Hepatitis C guidance: AASLDIDSA recommendations for testing, managing, and treating adults infected with hepatitis C virus. Hepatology 2015;62:932-954.

7. AbbVie Inc. VIEKIRA PAK. U.S. Food and Drug Administration web site, <https://www.accessdata.fda.gov/drugsatfda_docs/ label/2014/206619|bl.pdf>. Accessed 1 Dec 2014.

8. Abbie Ltd. New Zealand data sheet. New Zealand Medicines and Medical Devices Safety Authority web site, <http://www.medsafe. govt.nz/profs/datasheet/v/viekiraPaktab.pdf>. Accessed 16 Aug 2016.

9. Abdalla DA, Elhadidy TA, Besheer T, Farag RE. Respiratory adverse effects of sofosbuvir-based regimens for treatment of chronic hepatitis C virus. Egypt J Chest Dis Tuberc 2017;66:363-367.

10. Tamaki K, Okubo A. Simeprevir with peginterferon and ribavirin induced interstitial pneumonitis: first case report. World J Gastroenterol 2015;21:1009-1013.

11. Helmers RA, Byrne TJ, Wesselius LJ, Leslie KO. Serious pulmonary toxicity secondary to novel hepatitis $C$ antiviral therapy in a liver transplant recipient. Mayo Clin Proc 2015;90:1294-1297.

12. Slavenburg S, Heijdra YF, Drenth JP. Pneumonitis as a consequence of (peg)interferon-ribavirin combination therapy for hepatitis C: a review of the literature. Dig Dis Sci 2010;55:579-585.

13. Torres-Macho J, Jara-Chinarro B, Suárez-Aliaga B, Gámez-Díez S, García-de-Casasola G, de-Cuenca-Morón B. Pegylated interferon induced interstitial pneumonitis in a patient with hepatitis $C$ infection. Rev Esp Enferm Dig 2010;102:606-608.

14. Wu SY, Faire B, Gane E. Drug induced pneumonitis secondary to treatment with paritaprevir/ritonavir/ombitasvir and dasabuvir (VIEKIRA PAK(R)) for chronic hepatitis C: case report of an unexpected lifethreatening adverse reaction. Case Rep Med 2017;2017:4895736.

15. Tarao K, Yamada K. A hepatitis C-virus-associated cirrhotic patient developing interstitial pneumonia during the course of antiviral therapy with ombitasvir/paritaprevir/ritaonavir. Case Rep Gastroenterol 2017;11:367-376. 